\title{
HIV Infection and the Central Nervous System: A Primer
}

\author{
Ronald J. Ellis • Patricia Calero • Michael D. Stockin
}

Received: 20 February 2009 / Accepted: 22 April 2009/Published online: 5 May 2009

(C) The Author(s) 2009. This article is published with open access at Springerlink.com

\begin{abstract}
The purpose of this brief review is to prepare readers who may be unfamiliar with Human Immunodeficiency Virus/Acquired Immune Deficiency Syndrome (HIV/AIDS) and the rapidly accumulating changes in the epidemic by providing an introduction to HIV disease and its treatment. The general concepts presented here will facilitate understanding of the papers in this issue on HIV-associated neurocognitive disorders (HAND). Toward that end, we briefly review the biology of HIV and how it causes disease in its human host, its epidemiology, and how antiretroviral treatments are targeted to interfere with the molecular biology that allows the virus to reproduce. Finally, we describe what is known about how HIV injures the nervous system, leading to HAND, and discuss potential strategies for preventing or treating the effects of HIV on the nervous system.
\end{abstract}

Keywords HIV-associated neurocognitive disorders .

Central nervous system - Antiretroviral therapy .

HIV-associated injury · Nervous system repair .

Treatment of HAND

\section{HIV Biology and Pathogenesis}

Human immunodeficiency virus type 1 (HIV-1) principally infects a subgroup of immune cells called activated $\mathrm{CD} 4^{+}$

R. J. Ellis $\cdot$ P. Calero $\cdot$ M. D. Stockin

University of California, San Diego,

San Diego, CA, USA

R. J. Ellis $(\bowtie)$

HIV Neurobehavioral Research Center, University of California,

San Diego,

150 W. Washington Street, 2nd Floor,

San Diego, CA 92103, USA

e-mail: roellis@ucsd.edu lymphocytes, and secondarily infects macrophages, also a cellular component of the immune system. While infected macrophages are far fewer in number than lymphocytes, they are believed to be particularly important in the central nervous system (CNS). Mature viral particles feature a glycoprotein coat, or envelope, that allows them to identify, attach to, and enter these specific cell types.

At the earliest stage of its life cycle after infecting $\mathrm{CD}^{+}$ lymphocytes and macrophages, HIV directs these host cells to manufacture a viral enzyme, reverse transcriptase, which then converts viral RNA into DNA for insertion into the host cell's genetic library. HIV has no proofreading enzymes to correct for RNA to DNA conversion errors, leading to frequent DNA base substitutions and rapid genetic mutation. This propensity for copying errors is a major reason that HIV frequently develops resistance to therapy (see below). Furthermore, the fact that HIV DNA resides dormant, or replicatively inactive, in many cells protects it from detection by the immune system, making eradication of infection impossible even with the best available therapies.

HIV causes CNS disease in two general ways. Primary HIV CNS diseases are those for which the virus is both necessary and sufficient, while secondary CNS diseases require another, opportunistic pathogen that takes advantage of progressive immune deficiency. Although there is abundant evidence of primary HIV CNS injury, the virus does not directly infect neurons, and instead causes neuronal damage indirectly by infecting macrophages and other cells in the CNS.

\section{Disease Staging}

The clinical course of untreated HIV infection proceeds through four stages, which may or may not be apparent to 
patients themselves. The first stage is characterized by asymptomatic incubation lasting 2-4 weeks, followed by a symptomatic acute infection stage lasting 4 weeks on average (Kahn and Walker 1998). The symptoms of acute HIV infection are quite similar to those of many other viral infections, and therefore may pass without detection of HIV. Next follows a relatively silent period of ongoing viral replication and immune destruction known as the latency stage (Gottlieb et al. 2002). Its duration varies significantly, lasting anywhere from weeks to over a decade. During this time, patients may feel relatively well, again masking detection of the virus.

Left untreated, however, HIV almost always causes progressive immune system destruction, evidenced by drops in the numbers of $\mathrm{CD} 4^{+}$and $\mathrm{CD} 8^{+} \mathrm{T}$ cells (Laurence 1993). The result is acquired immune deficiency syndrome (AIDS), which is characterized by susceptibility to various opportunistic infections. AIDS is diagnosed when the immune system composition is altered below a threshold percentage or when T cell quantity is too low. According to the Centers for Disease Control's (CDC) 1993 standards, an HIV-infected individual has AIDS when either (a) their $\mathrm{CD}^{+}$cells fall to fourteen percent or less of total lymphocytes, or (b) when the $\mathrm{CD} 4^{+}$count is less than 200 cells $\mu \mathrm{L}$ or (c) when at least one of a specific set of opportunistic infections or neoplasms occurs (Centers for Disease Control and Prevention 1992). Current HIV treatment guidelines suggest starting combination antiretroviral therapy (cART) only in patients with advanced disease as defined by specific criteria that are revised on an annual basis (Hammer et al. 2008).

HIV replication is measured by quantifying the viral load, defined as the number of viral RNA copies per milliliter of blood plasma. Patients who maintain good adherence to cART often achieve reduction of the viral load to undetectable levels, curbing the destruction of $\mathrm{CD} 4^{+} \mathrm{T}$ lymphocytes (Gulick et al. 1997), but not eradicating the virus which reemerges in nearly all patients when cART is stopped. As these lymphocytes repopulate their numbers, the immune system regains strength. Thus cART frequently results in substantial immune reconstitution in HIV-infected individuals (Palella et al. 1998).

\section{Antiretroviral Therapy}

Since 1996, cART - also known as highly active antiretroviral therapy, or HAART - has produced dramatic improvements in health and longevity for HIV-infected individuals (Palella et al. 1998). cART options usually consist of three medications representing at least two different classes of antiretroviral drugs (see below). Multiple drugs from different classes are required because new mutations - including those that confer resistance to single drug or classes - arise at staggering rates. By inhibiting HIV replication at several stages in the viral life cycle, cART markedly reduces the likelihood that drug resistance will develop.

cART regimens typically include two nucleoside (or nucleotide) analogue reverse transcriptase inhibitors (NRTIs) and either a protease inhibitor or a nonnucleoside reverse transcriptase inhibitor (NNRTI). NRTIs and NNRTIs both interfere with reverse transcriptase function, preventing new viral particles from being formed. Nucleoside RTIs differ from their non-nucleoside counterparts in how they are designed to interfere with HIV reverse transcriptase. NRTIs interact with the chemically active binding site to prevent enzyme activity. However, NNRTIs bind to an allosteric regulation site, altering the transcriptase enzyme's shape and function. Protease inhibitors, the third component to most cART regimens, prevent the processing of manufactured viral particles through inhibition of HIV protease. Other classes of antiretroviral drugs include the integrase enzyme inhibitors, viral entry (fusion) inhibitors, and HIV maturation inhibitors. A regimen commonly prescribed to naïve patients is efavirenz (an NNRTI) plus tenofovir and emtricitabine (NRTIs) coformulated as a single pill given once a day, known as atripla.

Judicious use of antiretroviral therapy, by suppressing viral replication and partially restoring immune function, can prevent opportunistic infections and markedly prolong survival with HIV. As a result, most HIV brain disorders in the developed world today result not from opportunistic infections, but from primary HIV disease of the nervous system (Langford et al. 2003b).

\section{HIV Epidemiology}

According to estimates from the 2008 UNAIDS report on the global AIDS epidemic, there were approximately 33.2 million people living with HIV worldwide at the end of 2007 (Joint United Nations Programme on HIV/AIDS [UNAIDS] 2008). As prevention and treatment services have become more accessible, HIV-related deaths have declined - in 2007 there were 2.1 million HIV-related deaths worldwide, compared to 3.1 million in 2005 (UNAIDS 2008). These improvements in incidence and mortality have not been consistent throughout the world, occurring principally in developed countries. Although the HIV epidemic has stabilized somewhat since 2000 - in that the annual number of new HIV infections has declined from approximately 3.0 million in 2001 to 2.7 million in 2007the overall number of people living with HIV has steadily increased because new infections occur each year, and HIV treatment prolongs survival (UNAIDS 2008). 
While the majority of HIV research has taken place in Western countries, the virus itself is most prevalent and least-controlled in resource-limited settings such as subSaharan Africa and South and Southeast Asia (Centers for Disease Control and Prevention 2008; Sotrel and Dal Canto 2000). Although sub-Saharan Africa accounts for just over $10 \%$ of the world's population, the number of people living with HIV in this region at the end of 2007 was approximately 22 million, making sub-Saharan Africa home to $67 \%$ of all people living with HIV (UNAIDS 2007). AIDS remains the leading cause of death in this region, with $76 \%$ of all HIV-related deaths in 2007 occurring here (McCutchan 2006). In the same year, it is estimated that out of the 2.7 million new HIV infections worldwide, almost two thirds occurred in sub-Saharan Africa (UNAIDS 2007).

In Asia the number of people living with HIV at the end of 2007 was around 5.0 million, with the highest and fastest growing HIV infection levels concentrating in Southeast Asia (Centers for Disease Control and Prevention 2007). Epidemics in Indonesia, Pakistan, and Viet Nam are soaring. In Viet Nam alone, between 2000 and 2005, the number of people living with HIV more than doubled (UNAIDS 2007). Although sub-Saharan Africa remains the most seriously affected region by the AIDS epidemic, the most concerning increases in the incidence of new HIV infections are occurring in Eastern Europe and Central Asia. In this region, an estimated 1.6 million people were living with HIV at the end of 2007, an increase in prevalence of $150 \%$ since 2002 (UNAIDS 2007). Worst affected are Russia and Ukraine, with $62 \%$ of infections attributable to intravenous drug use (UNAIDS 2008).

In North America and Western and Central Europe there were approximately 2.0 million people living with HIV at the end of 2007-the United States accounting for an estimated 1.2 million of these (UNAIDS 2008).

Women account for half of all people living with HIV worldwide - at the end of 2007 an estimated 15.4 million women were infected with HIV (UNAIDS 2008). The proportion of women living with HIV varies significantly between different regions of the world, with a higher burden in sub-Saharan Africa and the Caribbean. For example, in sub-Saharan Africa women comprise nearly $61 \%$ of all adults living with HIV, while in the United States only $25 \%$ of people living with HIV are women (Centers for Disease Control and Prevention 2007; UNAIDS 2007). Over the last 10 years, the number of women living with HIV globally has remained stable, but in Latin America, Asia, and Eastern Europe this number is slowly increasing as they are infected by their male partners who are likely to have been infected through injecting drug use, or unprotected sex (UNAIDS 2007). In addition to the direct impact that HIV has on these women, there is also a known risk of mother-to-child transmission.

\section{HIV Subtypes}

HIV infections worldwide are characterized according to subtypes (clades), reflecting variants that are genetically more related to one another and often cluster in geographic regions because of founder effects. The high overall genetic diversity that characterizes HIV has been a key factor in its worldwide spread, and poses concerns for disease progression, as well as diagnosis, treatment and prevention efforts (Hemelaar et al. 2006). Findings from studies conducted in Western countries derive mainly from work on HIV-1 clade B infection in Caucasian populations, and therefore may not be generalizable to HIV clades and human populations globally. HIV-1 clade $\mathrm{B}$ is responsible for only $10 \%$ of infections worldwide and predominates in Western Europe, Australia, and the Americas (McCutchan 2006). In the United States, HIV1 clade B represents the vast majority of infections $(98 \%)$ with only minor contributions by other clades (Hemelaar et al.). However, in sub-Saharan Africa-the region most affected by the HIV epidemic-HIV-1 clade $\mathrm{C}$ is most common, accounting for about $50 \%$ of infections worldwide. In other areas heavily affected by the HIV epidemic, such as South and Southeast Asia, the predominant subtype is HIV-1 Clade AE. Finally, in Eastern Europe, Russia, and populous areas of Asia where the epidemic is rapidly expanding, 79\% of infections are caused by HIV-1 clade A (Hemelaar et al.).

The effects of HIV subtypes, or clades, on disease progression and treatment remain unclear (Liner et al. 2007). While numerous studies indicate that reverse transcriptase inhibitors and protease inhibitors are equally effective treating all HIV subtypes (Alexander et al. 2002; Bocket et al. 2005; Pillay et al. 2002), other studies claim that different HIV subtypes are more efficient in developing antiretroviral resistance, and present diminished drug sensitivity and HIV pathogenicity (Eshleman et al. 2001; Kantor et al. 2005; Spira et al. 2003).

\section{Mechanisms of HIV-Associated Injury}

HIV infection results in disruption of neuronal function by a variety of mechanisms that can be grouped into three general categories: viral factors, host factors and co-factors. Viral factors derive from the virus itself, and include several proteins encoded by the viral genome. Host factors, on the other hand, evolve indirectly or secondarily from infection, but may damage even 
uninfected cells such as neurons. Co-factors are social or behavioral characteristics or co-morbid conditions that may contribute to or amplify the pathogenicity of HIV. In the nervous system, these factors converge in producing damage to the elaborate network of connections between neurons that take place at dendrites and synapses. This synaptodendritic injury, described in detail below, disrupts the highly integrated functioning of neural systems that is required to process information, leading to HIV-associated neurocognitive disorders (HAND). As discussed in more detail in other manuscripts in this series, HIV associated dementia (HAD) is the most severe form of HAND. The brain, however, does not respond passively to synaptodendritic injury, but instead actively upregulates pathways promoting repair and regeneration. These pathways have become particularly important today, as survival has been prolonged by cART. Because both viral and host factors play a role in HAND, effective treatment might require both cART and so-called "adjunctive" therapies that include neuroprotective and neuroregenerative agents.

Neurotoxic Viral Factors The HIV genome codes for a variety of proteins that can damage neuronal cells and interfere with CNS function. Two of the more important viral proteins shown to be neurotoxic are gp120, the virus's envelope protein, and transactivator of transcription (Tat). gp120 is necessary for infectivity, but also interacts with host cellular receptors to alter glutamate pathway signaling and induce cytokine production that can injure neurons and affect the activation state of microglia and astrocytes. Patterns of neuronal injury seen in the brains of cognitively impaired HIV-infected individuals that come to autopsy can be replicated in cultures of human nervous tissue exposed to gp120 (Iskander et al. 2004) and in transgenic mice that express HIV-1 gp120 in the brain (Toggas et al. 1994). These changes include synaptodendritic injury, reactive astrocytosis, and microgliosis, and loss of large pyramidal neurons (Kaul and Lipton 2005). One molecular mechanism by which gp120 might induce these changes is through glutamate-mediated excitotoxicity, which can initiate caspase cascades (Tenneti and Lipton 2000).

Another viral protein reported to cause neuronal injury is Tat, which is produced by infected astrocytes (Nath 2002). In experiments in which Tat-expressing astrocytes were injected into the rat dentate gyrus, Tat was taken up by granule cells and transported along neuronal pathways to the CA3 region of the hippocampus, where it caused glial cell activation and neurotoxicity (Bruce-Keller et al. 2003). HIV Tat can cause mitochondrial dysfunction, dendritic loss, and cell death in neurons at concentrations lower than those needed to support viral replication (Chauhan et al. 2003).
Neurotoxic Host Factors Secondary effects of HIV infection on the immune system can amplify nervous system damage. Many of the host factors relevant to HIV CNS injury are chemical mediators of inflammation and immunity-cytokines and chemokines. Cytokines are produced by immune cells including macrophages that traffic into the CNS from the peripheral circulation, as well as by brain astrocytes and microglia activated or infected by HIV. Chemokines represent a subset of cytokines with chemoattractant properties, and are particularly important in HIVrelated pathology. Chemokine receptors are distributed throughout the brain on microglia, astrocytes, oligodendrocytes and neurons.

Abnormal activation of cytokine and chemokine receptors results in ultrastructural and functional neuronal alterations that are at least partially reversible. This suggests that appropriate treatments might enhance neuronal repair and ameliorate the damage arising from abnormal activation of cytokine or chemokine receptors. Importantly, host factors can differ between individuals. For example, increased susceptibility to neurodegeneration in HIV is associated with host genetic variations that might account for differential susceptibility to HAND between different individuals infected with the same viral strain (Gonzalez et al. 2002; Quasney et al. 2001).

Cofactors in CNS Injury HIV-infected individuals frequently have co-morbidities such as abuse of drugs and alcohol or infection with viral co-pathogens such as hepatitis $\mathrm{C}$ virus (HCV). These cofactors may contribute to CNS injury. For example, in neuropathological studies, HIVinfected individuals dying with histories of injection drug use show more activated microglia and diffuse astrogliosis in the white matter of the brain than their HIV-infected nonaddicted counterparts (Cook et al. 2005; Persidsky et al. 1996; Langford et al. 2003b) found that those dying with HIV and a history of methamphetamine addiction had greater loss of calbindin-immunostaining interneurons than in those with either condition alone. In vitro studies (Bagasra et al. 1993) as well as studies with rhesus monkeys (Tyor and Middaugh 1999) and mice (Winsauer et al. 2002) suggest that alcohol may exacerbate the immunological abnormalities associated with HIV infection, specifically the peripheral immune system. In addition, several studies have demonstrated that HCV co-infection was associated with a greater risk of neurocognitive disorders among those with HIV, with or without drug use (Cherner et al. 2004; Morgello et al. 2005). Because HCV is also associated with systemic and possibly CNS immune activation, it is possible that there may be some common immuno-neuropathogenic mechanisms leading to a heightened likelihood of CNS disease in co-infected individuals. 


\section{HIV-Associated Synaptodendritic Injury}

Synaptodendritic injury is a general term encompassing a variety of structural and chemical changes that ultimately can impair the normal functioning of neuronal networks. Normal synaptodendritic networks are complex and highly branching, whereas injured networks are simplified. Features of syndaptodendritic injury include dendritic spine retraction, beading and aberrant sprouting. Higher cognitive functions depend on the integrity of complex synaptodendritic networks, therefore damage results in deficient cognitive skills and behavior.

Synaptodendritic injury is demonstrated by immunostaining with antibodies to presynaptic synaptophysin (SYN) and postsynaptic microtubule associated protein2 (MAP2) (Orenstein et al. 1988). MAP2 is expressed on neuronal cell bodies and dendrites. In HIV-infected individuals, the degree of neurocognitive impairment is strongly related to loss of immunostaining for SYN and MAP2 (Moore et al. 2006). This technique has provided evidence that the striatum and the hippocampus are particularly affected (Bruce-Keller et al. 2003; Moore et al. 2006). This regional vulnerability parallels the higher burden of HIV proteins and viral RNA in the striatum and white matter connecting the striatum to the prefrontal cortex (Masliah et al. 1997).

Techniques for measuring synaptodendritic injury in living humans are indirect and imprecise. Proteins released from damaged neurons into the extracellular space can be detected in the cerebrospinal fluid (CSF) or blood. Elevated CSF neurofilament protein (NFL) concentrations, for example, are thought to reflect injury to myelinated axons. CSF NFL levels are increased both in the context of HIV dementia (Arnold 2001; Law et al. 2004) and after the interruption of cART, which results in a significant rebound of HIV replication. However, it is not known whether increased NFL can result from neuronal injury alone or requires cell death, and there are no data on the dynamic relationship between changes in neurological status in HIV patients and changes in NFL levels.

It is reasonable to propose that synaptodendritic loss will reduce the overall volume of brain gray and white matter. Gross structural atrophy can be visualized by brain computed tomography $(\mathrm{CT})$ and magnetic resonance imaging (MRI), and careful radiologicalneuropathological correlation studies have shown that white matter loss and abnormal white matter signal are closely correlated with the loss of MAP-2 immunostaining, particularly in the presence of HIV encephalitis (Archibald et al. 2004). Worsening white matter abnormalities correlate with worsening cognitive impairment (Everall et al. 1999).

\section{Nervous System Repair}

Synaptic dendritic networks in the healthy brain undergo continuous remodeling, reflecting plasticity, which can include increased dendritic branching, augmentation of axonal collaterals, generation of new synaptic connections and activity-dependent modification of existing synapses (Makrigeorgi-Butera et al. 1996). Various mediators of CNS plasticity may include brain derived neurotrophic factor (BDNF), insulin-like growth factor 1 (IGF-1), acidic fibroblast growth factor (aFGF, FGF1), macrophage inflammatory protein-2 (MIP2), stromal-derived factor1alpha (SDF-1 $\alpha$ ) and leptin (Isackson 1995; Pezet and Malcangio 2004; Shanley et al. 2001). Neurogenesis may also participate in restoration of function after brain injury (Langford et al. 2003a; Tomlinson et al. 1999).

\section{HIV-Associated Neurocognitive Disorders}

Neurodegeneration and synaptodendritic injury in HIV-infected individuals result in the clinical syndromes of HAND. Neurocognitive disorders can range in severity from slight deficits to debilitating dementia. In 2007 the National Institute of Mental Health (NIMH) and the National Institute of Neurological Diseases and Stroke (NINDS), commissioned the development of updated, working research criteria for HAND, based on criteria proposed by the San Diego HIV Neurobehavioral Research Center (HNRC). The resulting criteria defined three conditions: asymptomatic neurocognitive impairment (ANI), HIV-associated mild neurocognitive disorder (MND), and HIV-associated dementia (HAD) (Antinori et al. 2007). ANI describes individuals with usually mild impairment in two or more cognitive areas, demonstrated by neuropsychological testing, without a clear effect on everyday functioning. MND refers to the presence of mild to moderate deficits in two or more cognitive areas which create at least mild interference in everyday functioning. Finally, HAD describes individuals with documented moderate to severe deficits in two or more cognitive areas, with substantial impairment in every day functioning making the person incapable of employment and often unable to live independently.

Since the introduction of cART in 1996, the incidence of HAD, the more severe form of HAND, has decreased (McArthur 2004; McArthur et al. 1999). Despite this, the overall prevalence of HAND has not declined, possibly because HIV infected individuals with milder neurocognitive disorders are living longer, or because cART is not as effective in the brain as in the periphery. Thus milder impairments persist in many patients, with prevalence ranging between $30 \%$ and $50 \%$ depending in part upon disease stage, as documented in several reports in this volume. 


\section{Treatment of HAND}

The notion that cART might not be as effective in the brain as it is systemically derives from the observation that the blood brain barrier (BBB) frequently limits the movement of antiretrovirals from the circulation into the CNS. Several factors affect the CNS penetration of antiretroviral medications. Tight junctions between brain endothelial cells prevent the diffusion of polar molecules, making nonpolarity (lipophilicity) an important determinant of CNS penetration (Strazielle and Ghersi-Egea 2005). Highly protein-bound drugs have lower unbound concentrations, so fewer drug molecules are available to cross the BBB (Langford et al. 2006; Wynn et al. 2002). Once a drug does cross the BBB, efflux transporters - cellular proteins that pump various molecular substrates - may then promptly push it back into the circulation. As a result, HIV replication may persist in the CNS, despite adequate peripheral suppression (Blankson et al. 2002; De Luca et al. 2002; Wong et al. 1997).

The degree of penetration of antiretroviral therapy into the CNS seems to influence the extent of neurocognitive improvement, with individuals on regimens containing more CNS-penetrating drugs presenting better CSF viral load suppression (Letendre et al. 2004). Also, optimal antiretroviral concentrations in the CNS may be necessary to limit local HIV replication and prevent the development of drug-resistant viral strains in the CSF, which have the potential to re-infect the periphery (Reddy et al. 2003). Studies are ongoing to address the question of whether choosing antiretrovirals with greater CNS penetration will improve neurocognitive outcomes in HAND.

Beyond antiretroviral therapy, there is considerable interest in treating HAND with agents that promote neuronal repair or prevent further injury, so-called neuroprotective or regenerative therapies. For example, lithium, which is used to treat bipolar disorder, also modulates the expression of the pro-apoptotic glycogen synthase kinase $3 \beta$ (GSK $3 \beta$ ). In vitro, lithium prevents the induction of dendritic spine loss and simplification by HIV gp120 (Everall et al. 2002), indicating that it might be useful in HIV. In humans with HAND, lithium administration was associated with improved neurocognitive performance in a single-arm, 12-week study (Letendre et al. 2006). Minocycline is an antibiotic that has anti-inflammatory and neuroprotective effects that are currently being studied in humans. Memantine, a non-competitive antagonist of the NMDA receptor, prevents Tat and gp120-induced intracellular calcium increases and glutamate toxicity (Chen et al. 1998; Lipton and Chen 2004; Nath et al. 2000; Toggas et al. 1996). Early clinical trials suggest that some of these agents may have benefits, but none has been subjected to rigorous scientific evaluation in large, phase III licensing studies.

\section{Summary and Conclusions}

Understanding central nervous system injury in HIV infection requires an appreciation of some of the complexities of disease progression and its treatment. The outlook for patients with HIV has improved dramatically as increasingly effective antiretroviral therapies have become available. Nevertheless, neurocognitive impairment remains quite prevalent and is associated with reduced quality of life and social and occupational disabilities. Since HIV, with treatment, has become a chronic, manageable disease, the neurocognitive aspects of this condition should become an important focus for future research on pathogenesis and treatment.

Open Access This article is distributed under the terms of the Creative Commons Attribution Noncommercial License which permits any noncommercial use, distribution, and reproduction in any medium, provided the original author(s) and source are credited.

\section{References}

Alexander, C. S., Montessori, V., Wynhoven, B., Dong, W., Chan, K., O'Shaughnessy, M. V., et al. (2002). Prevalence and response to antiretroviral therapy of non-B subtypes of HIV in antiretroviral-naive individuals in British Columbia. Antiviral Therapy, 7(1), 31-35.

Antinori, A., Arendt, G., Becker, J. T., Brew, B. J., Byrd, D. A., Cherner, M., et al. (2007). Updated research nosology for HIVassociated neurocognitive disorders. Neurology, 69(18), 17891799. doi:10.1212/01.WNL.0000287431.88658.8b.

Archibald, S. L., Masliah, E., Fennema-Notestine, C., Marcotte, T. D., Ellis, R. J., McCutchan, J. A., et al. (2004). Correlation of in vivo neuroimaging abnormalities with postmortem human immunodeficiency virus encephalitis and dendritic loss. Archives of Neurology, 61(3), 369-376. doi:10.1001/archneur.61.3.369.

Arnold, S. E. (2001). Contributions of neuropathology to understanding schizophrenia in late life. Harvard Review of Psychiatry, 9(2), 69-76. doi:10.1080/10673220127882.

Bagasra, O., Kajdacsy-Balla, A., Lischner, H. W., \& Pomerantz, R. J. (1993). Alcohol intake increases human immunodeficiency virus type 1 replication in human peripheral blood mononuclear cells. The Journal of Infectious Diseases, 167(4), 789-797.

Blankson, J. N., Persaud, D., \& Siliciano, R. F. (2002). The challenge of viral reservoirs in HIV-1 infection. Annual Review of Medicine, 53, 557-593. doi:10.1146/annurev.med.53.082901.104024.

Bocket, L., Cheret, A., Deuffic-Burban, S., Choisy, P., Gerard, Y., de la Tribonniere, X., et al. (2005). Impact of human immunodeficiency virus type 1 subtype on first-line antiretroviral therapy effectiveness. Antiviral Therapy, 10(2), 247-254.

Bruce-Keller, A. J., Chauhan, A., Dimayuga, F. O., Gee, J., Keller, J. N., $\&$ Nath, A. (2003). Synaptic transport of human immunodeficiency virus-Tat protein causes neurotoxicity and gliosis in rat brain. The Journal of Neuroscience, 23(23), 8417-8422.

Centers for Disease Control and Prevention. (1992). 1993 revised classification system for HIV infection and expanded surveillance case definition for AIDS among adolescents and adults. Morvidity and Mortality Weekly Report Recommendations and Reports, 41(RR-17), 1-19. 
Centers for Disease Control and Prevention. (2007). HIV/AIDS surveillance report: Cases of HIV infection and AIDS in the United States and dependent areas, 2005. Atlanta, GA: Centers for Disease Control and Prevention.

Centers for Disease Control and Prevention. (2008). HIV/AIDS surveillance report, 2006. Atlanta, GA: United States Department of Health and Human Services.

Chauhan, A., Turchan, J., Pocernich, C., Bruce-Keller, A., Roth, S., Butterfield, D. A., et al. (2003). Intracellular human immunodeficiency virus tat expression in astrocytes promotes astrocyte survival but induces potent neurotoxicity at distant sites via axonal transport. The Journal of Biological Chemistry, 278, 13512-13519. doi:10.1074/jbc.M209381200.

Chen, H. S., Wang, Y. F., Rayudu, P. V., Edgecomb, P., Neill, J. C., Segal, M. M., et al. (1998). Neuroprotective concentrations of the N-methyl-D-aspartate open-channel blocker memantine are effective without cytoplasmic vacuolation following post-ischemic administration and do not block maze learning or long-term potentiation. Neuroscience, 86(4), 1121-1132. doi:10.1016/ S0306-4522(98)00163-8.

Cherner, M., Ellis, R. J., Lazzaretto, D., Young, C., Mindt, M. R., Atkinson, J. H., et al. (2004). Effects of HIV-1 infection and aging on neurobehavioral functioning: preliminary findings. AIDS (London, England), 18(Suppl. 1), S27-S34. doi:10.1097/ 00002030-200401001-00005.

Cook, J. E., Dasgupta, S., Middaugh, L. D., Terry, E. C., Gorry, P. R., Wesselingh, S. L., et al. (2005). Highly active antiretroviral therapy and human immunodeficiency virus encephalitis. Annals of Neurology, 57(6), 795-803. doi:10.1002/ana.20479.

De Luca, A., Ciancio, B. C., Larussa, D., Murri, R., Cingolani, A., Rizzo, M. G., et al. (2002). Correlates of independent HIV-1 replication in the CNS and of its control by antiretrovirals. Neurology, 59(3), 342-347.

Eshleman, S. H., Becker-Pergola, G., Deseyve, M., Guay, L. A., Mracna, M., Fleming, T., et al. (2001). Impact of human immunodeficiency virus type 1 (hiv-1) subtype on women receiving single-dose nevirapine prophylaxis to prevent hiv-1 vertical transmission (hiv network for prevention trials 012 study). The Journal of Infectious Diseases, 184(7), 914-917. doi:10.1086/323153.

Everall, I. P., Heaton, R. K., Marcotte, T. D., Ellis, R. J., McCutchan, J. A., \& Atkinson, J. H. (1999). Cortical synaptic density is reduced in mild to moderate human immunodeficiency virus neurocognitive disorder. HNRC Group. The HIV Neurobehavioral Research Center. Brain Pathology (Zurich, Switzerland), 9(2), 209-217.

Everall, I. P., Bell, C., Mallory, M., Langford, D., Adame, A., Rockestein, E., et al. (2002). Lithium ameliorates HIV-gp120mediated neurotoxicity. Molecular and Cellular Neurosciences, 21(3), 493-501. doi:10.1006/mone.2002.1196.

Gonzalez, E., Rovin, B. H., Sen, L., Cooke, G., Dhanda, R., Mummidi, S., et al. (2002). HIV-1 infection and AIDS dementia are influenced by a mutant MCP-1 allele linked to increased monocyte infiltration of tissues and MCP-1 levels. Proceedings of the National Academy of Sciences of the United States of America, 99(21), 13795-13800. doi:10.1073/ pnas.202357499.

Gottlieb, G. S., Sow, P. S., Hawes, S. E., Ndoye, I., Redman, M., CollSeck, A. M., et al. (2002). Equal plasma viral loads predict a similar rate of $\mathrm{CD} 4+\mathrm{T}$ cell decline in human immunodeficiency virus (HIV) type 1- and HIV-2-infected individuals from Senegal, West Africa. The Journal of Infectious Diseases, 185(7), 905914. doi:10.1086/339295.

Gulick, R. M., Mellors, J. W., Havlir, D., Eron, J. J., Gonzalez, C., McMahon, D., et al. (1997). Treatment with indinavir, zidovudine, and lamivudine in adults with human immunodeficiency virus infection and prior antiretroviral therapy. The New
England Journal of Medicine, 337(11), 734-739. doi:10.1056/ NEJM199709113371102.

Hammer, S. M., Eron, J. J., Jr., Reiss, P., Schooley, R. T., Thompson, M. A., Walmsley, S., et al. (2008). Antiretroviral treatment of adult HIV infection: 2008 recommendations of the International AIDS Society-USA panel. Journal of the American Medical Association, 300(5), 555-570. doi:10.1001/ jama.300.5.555.

Hemelaar, J., Gouws, E., Ghys, P. D., \& Osmanov, S. (2006). Global and regional distribution of HIV-1 genetic subtypes and recombinants in 2004. AIDS (London, England), 20, W13W23. doi:10.1097/01.aids.0000247564.73009.bc.

Isackson, P. J. (1995). Trophic factor response to neuronal stimuli or injury. Current Opinion in Neurobiology, 5(3), 350-357. doi:10.1016/ 0959-4388(95)80048-4.

Iskander, S., Walsh, K. A., \& Hammond, R. R. (2004). Human CNS cultures exposed to HIV-1 gp120 reproduce dendritic injuries of HIV-1-associated dementia. Journal of Neuroinflammation, 1(1), 7. doi:10.1186/1742-2094-1-7.

Kahn, J. O., \& Walker, B. D. (1998). Acute human immunodeficiency virus type 1 infection. The New England Journal of Medicine, 339(1), 33-39. doi:10.1056/NEJM199807023390107.

Kantor, R., Katzenstein, D. A., Efron, B., Carvalho, A. P., Wynhoven, B., Cane, P., et al. (2005). Impact of HIV-1 subtype and antiretroviral therapy on protease and reverse transcriptase genotype: results of a global collaboration. PLoS Medicine, 2 (4), e112. doi:10.1371/journal.pmed.0020112.

Kaul, M., \& Lipton, S. A. (2005). Experimental and potential future therapeutic approaches for HIV-1 associated dementia targeting receptors for chemokines, glutamate and erythroprotein. Neurotoxicity Research, 8, 167-186.

Langford, D., Adame, A., Grigorian, A., Grant, I., McCutchan, J. A., Ellis, R. J., et al. (2003a). Patterns of selective neuronal damage in methamphetamine-user AIDS patients. Journal of Acquired Immune Deficiency Syndromes, 34(5), 467-474. doi:10.1097/00126334200312150-00004.

Langford, T. D., Letendre, S. L., Larrea, G. J., \& Masliah, E. (2003b). Changing patterns in the neuropathogenesis of HIV during the HAART era. Brain Pathology (Zurich, Switzerland), 13(2), 195-210.

Langford, D., Marquie-Beck, J., de Almeida, S., Lazzaretto, D., Letendre, S., Grant, I., et al. (2006). Relationship of antiretroviral treatment to postmortem brain tissue viral load in human immunodeficiency virus-infected patients. Journal of Neurovirology, 12(2), 100-1007. doi:10.1080/13550280600713932.

Laurence, J. (1993). T-cell subsets in health, infectious disease, and idiopathic CD4+ T lymphocytopenia. Annals of Internal Medicine, 119(1), 55-62.

Law, A. J., Weickert, C. S., Hyde, T. M., Kleinman, J. E., \& Harrison, P. J. (2004). Reduced spinophilin but not microtubule-associated protein 2 expression in the hippocampal formation in schizophrenia and mood disorders: molecular evidence for a pathology of dendritic spines. The American Journal of Psychiatry, 161(10), 1848-1855. doi:10.1176/appi. ajp. 161.10.1848.

Letendre, S. L., McCutchan, J. A., Childers, M. E., Woods, S. P., Lazzaretto, D., Heaton, R. K., et al. (2004). Enhancing antiretroviral therapy for human immunodeficiency virus cognitive disorders. Annals of Neurology, 56(3), 416-423. doi:10.1002/ana.20198.

Letendre, S. L., Woods, S. P., Ellis, R. J., Atkinson, J. H., Masliah, E., van den Brande, G., et al. (2006). Lithium improves HIVassociated neurocognitive impairment. AIDS (London, England, 20(14), 1885-1888. doi:10.1097/01.aids.0000244208.49123.1b.

Liner, K. J., 2nd, Hall, C. D., \& Robertson, K. R. (2007). Impact of human immunodeficiency virus (HIV) subtypes on HIVassociated neurological disease. Journal of Neurovirology, 13 (4), 291-304. doi:10.1080/13550280701422383. 
Lipton, S. A., \& Chen, H. S. (2004). Paradigm shift in neuroprotective drug development: clinically tolerated NMDA receptor inhibition by memantine. Cell Death and Differentiation, 11(1), 18-20. doi:10.1038/sj.cdd.4401344.

Makrigeorgi-Butera, M., Hagel, C., Laas, R., Puschel, K., \& Stavrou, D. (1996). Comparative brain pathology of HIV-seronegative and HIVinfected drug addicts. Clinical Neuropathology, 15(6), 324-329.

Masliah, E., Heaton, R. K., Marcotte, T. D., Ellis, R. J., Wiley, C. A., Mallory, M., et al. (1997). Dendritic injury is a pathological substrate for human immunodeficiency virus-related cognitive disorders. HNRC Group. The HIV Neurobehavioral Research Center. Annals of Neurology, 42(6), 963-972. doi:10.1002/ ana.410420618.

McArthur, J. C. (2004). HIV dementia: an evolving disease. Journal of Neuroimmunology, 157(1-2), 3-10. doi:10.1016/j. jneuroim.2004.08.042.

McArthur, J. C., Sacktor, N., \& Selnes, O. (1999). Human immunodeficiency virus-associated dementia. Seminars in Neurology, 19(2), 129-150. doi:10.1055/s-2008-1040831.

McCutchan, F. E. (2006). Global epidemiology of HIV. Journal of Medical Virology, 78(Suppl), 7-12. doi:10.1002/jmv.20599.

Moore, D. J., Masliah, E., Rippeth, J. D., Gonzalez, R., Carey, C. L., Cherner, M., et al. (2006). Cortical and subcortical neurodegeneration is associated with HIV neurocognitive impairment. AIDS (London, England), 20(6), 879-887. doi:10.1097/01. aids.0000218552.69834.00.

Morgello, S., Estanislao, L., Ryan, E., Gerits, P., Simpson, D., Verma, S., et al. (2005). Effects of hepatic function and hepatitis C virus on the nervous system assessment of advanced-stage HIVinfected individuals. AIDS (London, England), 19(Suppl. 3), S116-S122. doi:10.1097/01.aids.0000192079.49185.f9.

Nath, A. (2002). Human immunodeficiency virus (HIV) proteins in neuropathogenesis of HIV dementia. The Journal of Infectious Diseases, 186, S193-S198. doi:10.1086/344528.

Nath, A., Haughey, N. J., Jones, M., Anderson, C., Bell, J. E., \& Geiger, J. D. (2000). Synergistic neurotoxicity by human immunodeficiency virus proteins Tat and gp120: protection by memantine. Annals of Neurology, 47(2), 186-194. doi:10.1002/ 1531-8249(200002) 47:2\&1t;186::AID-ANA8\&gt;3.0.CO;2-3.

Orenstein, J. M., Meltzer, M. S., Phipps, T., \& Gendelman, H. E. (1988). Cytoplasmic assembly and accumulation of human immunodeficiency virus types 1 and 2 in recombinant human colony-stimulating factor-1-treated human monocytes: an ultrastructural study. Journal of Virology, 62(8), 2578-2586.

Palella, F. J., Jr., Delaney, K. M., Moorman, A. C., Loveless, M. O., Fuhrer, J., Satten, G. A., et al. (1998). Declining morbidity and mortality among patients with advanced human immunodeficiency virus infection. HIV Outpatient Study Investigators. The New England Journal of Medicine, 338(13), 853-860. doi:10.1056/ NEJM199803263381301.

Persidsky, Y., Limoges, J., McComb, R., Bock, P., Baldwin, T., Tyor, W., et al. (1996). Human immunodeficiency virus encephalitis in SCID mice. American Journal of Pathology, 149(3), 1027-1053.

Pezet, S., \& Malcangio, M. (2004). Brain-derived neurotrophic factor as a drug target for CNS disorders. Expert Opinion on Therapeutic Targets, 8(5), 391-399. doi:10.1517/14728222.8.5.391.

Pillay, D., Walker, A. S., Gibb, D. M., de Rossi, A., Kaye, S., Ait-Khaled, M., et al. (2002). Impact of human immunodeficiency virus type 1 subtypes on virologic response and emergence of drug resistance among children in the Paediatric European Network for Treatment of AIDS (PENTA) 5 trial. The Journal of Infectious Diseases, 186(5), 617-625. doi: $10.1086 / 342680$.
Quasney, M. W., Zhang, Q., Sargent, S., Mynatt, M., Glass, J., \& McArthur, J. (2001). Increased frequency of the tumor necrosis factor- $\alpha-308$ a allele in adults with human immunodeficiency virus dementia. Annals of Neurology, 50(2), 157-162. doi:10.1002/ana.1284.

Reddy, Y. S., Kashuba, A., Gerber, J., \& Miller, V. (2003). Roundtable report: importance of antiretroviral drug concentrations in sanctuary sites and viral reservoirs. AIDS Research and Human Retroviruses, 19(3), 167-176. doi:10.1089/ 088922203763315669.

Shanley, L. J., Irving, A. J., \& Harvey, J. (2001). Leptin enhances NMDA receptor function and modulates hippocampal synaptic plasticity. The Journal of Neuroscience, 21(24), RC186.

Sotrel, A., \& Dal Canto, M. C. (2000). HIV-1 and its causal relationship to immunosuppression and nervous system disease in AIDS: a review. Human Pathology, 31, 1274-1298. doi:10.1053/hupa.2000.19293.

Spira, S., Wainberg, M. A., Loemba, H., Turner, D., \& Brenner, B. G. (2003). Impact of clade diversity on HIV-1 virulence, antiretroviral drug sensitivity and drug resistance. The Journal of Antimicrobial Chemotherapy, 51, 229-240. doi:10.1093/jac/dkg079.

Strazielle, N., \& Ghersi-Egea, J. F. (2005). Factors affecting delivery of antiviral drugs to the brain. Reviews in Medical Virology, 15 (2), 105-133. doi:10.1002/rmv.454.

Tenneti, L., \& Lipton, S. A. (2000). Involvement of activated caspase3 -like proteases in $\mathrm{N}$-methyl-D-aspartate-induced apoptosis in cerebrocortical neurons. Journal of Neurochemistry, 74, 134142. doi:10.1046/j.1471-4159.2000.0740134.x.

Toggas, S. M., Masliah, E., Rockenstein, E. M., Rail, G. F., Abraham, C. R., \& Mucke, L. (1994). Central nervous system damage produced by expression of the HIV-1 coat protein gp120 in transgenic mice. Nature, 367(6459), 188-193. doi:10.1038/367188a0.

Toggas, S. M., Masliah, E., \& Mucke, L. (1996). Prevention of HIV-1 gp120-induced neuronal damage in the central nervous system of transgenic mice by the NMDA receptor antagonist memantine. Brain Research, 706(2), 303-307. doi:10.1016/ 0006-8993(95)01197-8.

Tomlinson, G. S., Simmonds, P., Busuttil, A., Chiswick, A., \& Bell, J. E. (1999). Upregulation of microglia in drug users with and without pre-symptomatic HIV infection. Neuropathology and Applied Neurobiology, 25(5), 369-379. doi:10.1046/j.13652990.1999.00197.x.

Tyor, W. R., \& Middaugh, L. D. (1999). Do alcohol and cocaine abuse alter the course of HIV-associated dementia complex? Journal of Leukocyte Biology, 65(4), 475-481.

UNAIDS Joint United Nations Programme on HIV/AIDS. (2007). AIDS epidemic update. (Report). Geneva: Joint United Nations Programme on HIV/AIDS.

UNAIDS. Joint United Nations Programme on HIV/AIDS. (2008). Report on the global AIDS epidemic. Geneva, Switzerland: Joint United Nations Programme on HIV/AIDS.

Winsauer, P. J., Moerschbaecher, J. M., Brauner, I. N., Purcell, J. E., Lancaster, J. R., Jr., Bagby, G. J., et al. (2002). Alcohol unmasks simian immunodeficiency virus-induced cognitive impairments in rhesus monkeys. Alcoholism, Clinical and Experimental Research, 26(12), 1846-1857.

Wong, J. K., Hezareh, M., Gunthard, H. F., Havlir, D. V., Ignacio, C. C., Spina, C. A., et al. (1997). Recovery of replicationcompetent HIV despite prolonged suppression of plasma viremia. Science, 278(5341), 1291-1295. doi:10.1126/ science.278.5341.1291.

Wynn, H. E., Brundage, R. C., \& Fletcher, C. V. (2002). Clinical implications of CNS penetration of antiretroviral drugs. CNS Drugs, 16, 595-609. doi:10.2165/00023210-200216090-00002. 